\title{
Exclusive olive oil consumption has a protective effect on coronary artery disease; overview of the THISEAS study
}

\author{
Maria Dimitriou ${ }^{1, *}$, Loukianos S Rallidis ${ }^{2}$, Eirini $\bigvee$ Theodoraki ${ }^{1}$, loanna Panagiota Kalafati ${ }^{1}$, \\ Genovefa Kolovou ${ }^{3}$ and George V Dedoussis ${ }^{1, *}$ \\ 'Department of Nutrition and Dietetics, School of Health Science and Education, Harokopio University, El. Venizelou \\ 70, 17671 Athens, Greece: ${ }^{2}$ Second Department of Cardiology, Attikon Hospital, School of Medicine, University of \\ Athens, Athens, Greece: ${ }^{3}$ First Cardiology Department, Onassis Cardiac Surgery Center, Athens, Greece
}

Submitted 25 March 2015: Final revision received 8 June 2015: Accepted 24 June 2015: First published online 30 July 2015

\begin{abstract}
Objective: The aims of the current report are to present the demographic characteristics, clinical characteristics/biochemical indices and lifestyle habits of the population and to explore the potential association of exclusive olive oil consumption, in relation to lifestyle factors, with coronary artery disease risk.

Design: Demographic, lifestyle, dietary and biochemical variables were recorded. Logistic regression analysis was performed in order to estimate the relative risks of developing coronary artery disease.

Setting: The Hellenic study of Interactions between Single nucleotide polymorphisms and Eating in Atherosclerosis Susceptibility (THISEAS), a medical centre-based case-control study conducted in Greek adults.

Subjects: We consecutively enrolled 1221 adult patients with coronary artery disease and 1344 adult controls.

Results: A higher prevalence of the conventional established risk factors was observed in cases than in controls. Physical activity level was higher in controls (1.4 (SD 0.2) than in cases (1.3 (SD 0.3); $P<0.001)$. Regarding current and ex-smokers, the case group reported almost double the pack-years of the control group (54.6 (sD 42.8) v. 28.3 (SD 26.3), respectively; $P<0 \cdot 001$ ). Exclusive olive oil consumption was associated with $37 \%$ lower likelihood of developing coronary artery disease, even after taking into account adherence to the Mediterranean diet (OR $=0.63 ; 95 \%$ CI $0.42,0.93 ; P=0.02$ ).

Conclusions: Exclusive olive oil consumption was associated with lower risk of coronary artery disease, even after adjusting for adoption of an overall healthy dietary pattern such as the Mediterranean diet.
\end{abstract}

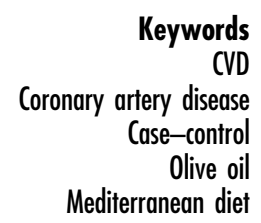

Coronary artery disease (CAD) is the most common disorder of CVD. It causes narrowing of the lumen of one or more of the coronary arteries, resulting in angina pectoris, myocardial infarction or congestive heart failure. It is estimated that 17.3 million people died from CVD in 2008 and this number contributes $30 \%$ of all deaths worldwide. Of these deaths, $7 \cdot 3$ million were due to $\mathrm{CAD}^{(1)}$.

In Greece, the prevalence of CVD risk factors was originally examined by the Seven Countries Study ${ }^{(2)}$. That study paved the way for other observational studies to follow, which measured the prevalence of CVD risk factors at population level ${ }^{(3-5)}$. The results of these studies have mainly highlighted that dislipidaemia, hypertension, obesity and environmental factors such as unhealthy dietary habits, smoking, physical inactivity and stress contribute to the development of CAD. Among the established environmental factors, dietary habits and their relationship to CAD have gained the attention of many scientists for more than half a century. The Seven Countries study was again the first to highlight a dietary pattern, namely the Mediterranean diet (MedDiet), as cardioprotective $^{(6-8)}$. Along the same lines, other studies have consistently reported that adherence to this type of dietary pattern is beneficial against cardiovascular risk $^{(9,10)}$. It has been proposed that the MedDiet exerts a beneficial influence against $\mathrm{CAD}$ risk factors and $\mathrm{CAD}$ occurrence mainly due to the abundant consumption of olive oil rich in MUFA ${ }^{(11)}$. Many studies have demonstrated an inverse relationship between olive oil consumption and the risk of $\mathrm{CVD}^{(12,13)}$. Moreover, as obesity rates are rising and lipid profiles are deteriorating within the Greek population, total fat intake, SFA intake and levels of physical activity should also be considered before advising for a Mediterranean food pattern adoption 
independently of the fat content ${ }^{(14)}$. Therefore, more evidence is needed to elucidate the association between olive oil consumption, in the context of MedDiet adoption, and CAD risk. Furthermore, even more scarce data exist regarding the effect of exclusive olive oil consumption on CAD risk.

The Hellenic study of Interactions between Single nucleotide polymorphisms and Eating in Atherosclerosis Susceptibility (THISEAS) is a medical centre-based casecontrol study. The aims of the current report are to: (i) present the demographic characteristics, clinical characteristics/biochemical indices and lifestyle habits of the Greek sample; and (ii) explore the potential association of exclusive olive oil consumption, in relation to lifestyle factors, with CAD risk.

\section{Materials and methods}

\section{Study population}

Study participants were consecutively recruited from (i) major hospitals, (ii) Centers of Open Protection for the Elderly and (iii) municipalities, all located in the region of Athens.

Controls were either out-patients for routine examinations or in-patients in departments other than cardiology clinics, who visited the same hospitals and during the same period as the coronary patients. Healthy volunteers from Centers of Open Protection for the Elderly and municipalities of the area of Athens were also enrolled in the control group. In order to reduce the effect of possible unknown confounders and to eliminate the problem of misclassification, we retrieved precise information from medical history through the hospital or insurance records. Therefore, controls were individuals with negative coronary angiography findings or a negative stress test, or individuals without clinical symptoms of CAD, any CVD, cancer or inflammatory diseases.

Cases were enrolled from cardiology clinics of Athens' hospitals. Cases were individuals presenting with either acute coronary syndrome or stable CAD defined as > $50 \%$ stenosis in at least one of the three main coronary vessels assessed by coronary angiography. Acute coronary syndrome was defined as acute myocardial infarction ranging from ST-segment (STEMI) to non-ST-segment elevation (NSTEMI) and unstable angina ${ }^{(15)}$. Acute coronary syndrome patients had also undergone coronary angiography that verified the presence of significant stenosis. Individuals with renal or hepatic disease were excluded from both study groups. In the group of cases, $60.4 \%$ were diagnosed with first-time CAD at the time of recruitment, while $39.6 \%$ were diagnosed with $\mathrm{CAD}$ six months or more before the time of recruitment.

All participants were informed about the goals of the study and gave their written consent. A total of 2886 people were invited to participate in the study and 2565 were finally recruited from 2006 to 2010.
The study protocol was approved by the Ethics Committee of Harokopio University.

\section{Blood sampling and analysis}

Blood samples were collected between 06.00 and 10.00 hours after a $12 \mathrm{~h}$ fast. Blood analysis included haematological and biochemical indices: fasting glucose, total cholesterol (TC), HDL cholesterol (HDL-C) and TAG. LDL cholesterol (LDL-C) was calculated from the Friedewald equation $^{(16)}$. Furthermore, plasma and serum were isolated and stored at $-80^{\circ} \mathrm{C}$ for future measurements.

\section{Demographic, anthropometric and lifestyle assessments}

Demographic and lifestyle characteristics, personal and family medical history of CVD risk factors, dietary habits and physical activity habits were assessed during recruitment of the study sample.

All participants were interviewed regarding their origins to ensure their Greek ancestry. Their educational status was measured by the years of schooling and was classified into four groups: (i) primary school (0-6 years); (ii) high school (7-12 years); (iii) senior high or technical institutes (13-15 years); and (iv) university ( $\geq 16$ years). Senior high or technical institutes and university were combined into one group. The marital status of the participants was also recorded and defined as single, married, divorced or widowed. The annual financial status of the participants was classified into four groups: (i) low $(<8000 €)$; (ii) medium (8000-15000€); (iii) high (15000-20 $000 €)$; and (iv) very high (>20 $000 €$ ). Low and medium financial status were combined into one group (low/medium). Similarly, high and very high financial status were combined into one group (high/very high). Furthermore, data regarding professional status was collected and the participants were accordingly characterized as public servants, private employees, freelancers, partially employed, unemployed, retired and householders.

Current smokers were defined as those who smoked at least one cigarette daily, non-smokers as those who have never smoked in their life and former smokers as those who have stopped smoking for at least six months. Pack-years (cigarette packs per day $\times$ years of smoking) were also calculated.

\section{Anthropometric and clinical assessments}

A physician performed a clinical assessment of the participants through a questionnaire during an interview. In order to eliminate recall bias, we also tried to reclaim medical information from the hospital or insurance records for both study groups, where available. Hypercholesterolaemia was defined as TC level greater than $200 \mathrm{mg} / \mathrm{dl}$ or use of lipid-lowering medication. Diabetics were those with blood glucose level greater than $125 \mathrm{mg} / \mathrm{dl}$ or individuals under a special diet or treatment. 
Blood pressure level was measured in the right arm, with the participant seated and rested, using a mercury sphygmomanometer. Hypertension was defined as blood pressure greater than $140 / 90 \mathrm{mmHg}$ or use of antihypertensive medication.

Family history of premature CAD was considered when the age at which first-degree relatives developed symptomatic CAD was $<55$ years for males and $<65$ years for females.

Finally, body weight and height were measured for all participants (wearing light clothing, without shoes) using a levelled platform scale and a wall-mounted stadiometer, to the nearest $0.5 \mathrm{~kg}$ and $0.5 \mathrm{~cm}$, respectively. BMI was computed as [weight $(\mathrm{kg})] /[\text { height }(\mathrm{m})]^{2}$.

\section{Physical activity assessment}

Physical activity level (PAL) was assessed through the Harokopio Physical Activity Questionnaire (HAPAQ) ${ }^{(17)}$ that evaluates the frequency, duration and intensity of occupational, household and leisure-time activities. PAL was calculated as the ratio of the work metabolic rate to the resting metabolic rate of 1 metabolic equivalent (MET). The work metabolic rate was defined as the person's total energy expenditure in a $24 \mathrm{~h}$ period, based on performed physical activities. The caloric cost of each physical activity (PA) was estimated with the equation: kilocalories $=$ MET $\times$ weight in kilograms $\times$ duration in hours. The 1 MET reference value of $1 \mathrm{kcal} / \mathrm{kg}$ per $\mathrm{h}$ is used by convention and refers to a metabolic rate at rest of an average individual $^{(18)}$. PA adoption was assessed as a categorical variable categorizing the participants into two groups, whether they reported leisure-time activities on a regular basis or not.

\section{Dietary assessment}

Nutritional information was obtained from $60 \cdot 4 \%(n$ 737) of patients who were first diagnosed with CAD within six months by the time of their recruitment into the study. The volunteers were asked about their nutritional habits before CAD diagnosis. Nutritional information from participants with CAD diagnosis for more than six months at the time of recruitment was not collected, in order to eliminate: (i) bias regarding possible modifications of nutritional habits after CAD diagnosis; and (ii) recall bias regarding nutritional habits before CAD diagnosis.

We tried to retrieve dietary information from double the number of controls, in order to ensure a controls-to-cases ratio of 2:1. After excluding individuals with incomplete or missing dietary data and extreme values of energy intake, the main sample with nutritional information included 499 cases and 832 controls.

Dietary data were collected through a 172 -item picturesort FFQ. Participants were asked to indicate how often they consumed various foods and beverages, as well as the portion size by comparison with photos. Daily consumption was calculated from the FFQ by multiplying the standard serving size of each food (as described by the Ministry of Health and Welfare, Supreme Scientific Health Council) by the value corresponding to each consumption frequency: never; 1-3 times/month; 1-2 times/week; 3-4 times/week; 5-6 times/week; and 1 time/ $\mathrm{d}^{(19)}$. The FFQ was either self-administered or self-interviewed. In both cases, well-trained nutritional professionals were available to assist the participants during questionnaire completion.

Nutritionist Pro version 2.2 software (Axxya SystemsNutritionist Pro, Stafford, TX, USA) was used to analyse nutritional information regarding energy, macronutrient and micronutrient intakes.

\section{Mediterranean diet score evaluation}

Adherence to the MedDiet was evaluated by calculating a MedDietScore (MDS) that is an a priori-defined score. The range of the MDS is between 0 and 55 ; higher values suggest greater adherence to the traditional Mediterranean dietary pattern that is characterized by high consumption of plant foods (non-refined cereals, vegetables and fruits) and olive oil, by moderate consumption of dairy products, poultry and fish, and by low consumption of meat and meat products ${ }^{(20)}$. The MDS was split at the median into two categories to indicate the participants with low adherence (below the median) and high adherence (above the median) to the MedDiet.

\section{Evaluation of olive oil consumption}

The FFQ included additional questions to assess the type of fat/oil used in cooking. Participants were asked to report the type of fat/oil used during the preparation of: (i) salads; (ii) cooked meals; and (iii) fried meals. Each participant could indicate more than one choice of fat/oil, namely olive oil, seed oil, corn oil, sunflower oil, butter and margarine, as depicted in the online supplementary material, Supplemental Table 1. Regarding olive oil consumption, participants were classified into two groups: (i) those who exclusively used olive oil during meal and salad preparation; and (ii) those who consumed other types of fat/oil as well.

\section{Statistical analysis}

Continuous variables are presented as mean values and standard deviations, while categorical variables are presented as frequencies. Differences between categorical variables and groups of the study were assessed using the $\chi^{2}$ test. P-P plots were applied to assess the normality of the distribution of the continuous variables. Student's $t$ test or the Mann-Whitney test was applied to evaluate differences in continuous variables between the two study groups. Logistic regression analysis was performed in order to estimate the relative risks of developing CAD by the calculation of odds ratios and their corresponding $95 \%$ confidence intervals. All reported $P$ values are based on two-sided tests and compared with a significance level of $5 \%$. The statistical software package IBM SPSS Statistics 21.0 was used for all statistical calculations. 
Table 1 Sociodemographic characteristics of the study participants; THISEAS study, 2006-2010.

\begin{tabular}{|c|c|c|c|c|c|}
\hline & \multicolumn{2}{|c|}{$\begin{array}{l}\text { CAD patients } \\
\quad(n 1221)\end{array}$} & \multicolumn{2}{|c|}{$\begin{array}{l}\text { Controls } \\
(n 1344)\end{array}$} & \multirow[b]{2}{*}{$\begin{array}{c}P \\
\text { value }^{*}\end{array}$} \\
\hline & $\begin{array}{l}\text { Mean or } \\
\text { frequency }\end{array}$ & SD & $\begin{array}{l}\text { Mean or } \\
\text { frequency }\end{array}$ & SD & \\
\hline Age (years) & $62 \cdot 8$ & $10 \cdot 4$ & $57 \cdot 7$ & $14 \cdot 1$ & $<0.001$ \\
\hline Male sex (\%) & $81 \cdot 8$ & - & $46 \cdot 3$ & - & $<0.001$ \\
\hline Educational status (\%) & $10 \cdot 5$ & $4 \cdot 8$ & $11 \cdot 5$ & $4 \cdot 7$ & $<0.001$ \\
\hline Primary school & $36 \cdot 7$ & - & $28 \cdot 1$ & - & $<0.001$ \\
\hline High school & $34 \cdot 2$ & - & $34 \cdot 1$ & - & \\
\hline $\begin{array}{l}\text { Tertiary education/ } \\
\text { university }\end{array}$ & $29 \cdot 1$ & - & $37 \cdot 8$ & - & \\
\hline \multicolumn{6}{|l|}{ Profession (\%) } \\
\hline Public servants & $7 \cdot 9$ & - & $17 \cdot 8$ & - & $<0.001$ \\
\hline Private employees & $10 \cdot 1$ & - & $18 \cdot 2$ & - & \\
\hline Freelancers & $19 \cdot 2$ & - & $14 \cdot 6$ & - & \\
\hline Partially employed & $0 \cdot 2$ & - & $1 \cdot 4$ & - & \\
\hline Unemployed & 0.3 & - & 4.9 & - & \\
\hline Retired & $54 \cdot 3$ & - & $33 \cdot 1$ & - & \\
\hline Householders & $8 \cdot 0$ & - & $10 \cdot 0$ & - & \\
\hline \multicolumn{6}{|l|}{ Annual income (\%) } \\
\hline Low/medium & $44 \cdot 3$ & - & $38 \cdot 1$ & - & $<0.001$ \\
\hline High/very high & $55 \cdot 7$ & - & 61.9 & - & \\
\hline \multicolumn{6}{|l|}{ Marital status (\%) } \\
\hline Single & $21 \cdot 8$ & - & $12 \cdot 3$ & - & \\
\hline Married & $69 \cdot 7$ & - & $66 \cdot 4$ & - & $<0.05$ \\
\hline Divorced & $5 \cdot 3$ & - & $15 \cdot 6$ & - & \\
\hline Widow/er & $3 \cdot 2$ & - & $5 \cdot 8$ & - & \\
\hline
\end{tabular}

THISEAS, The Hellenic study of Interactions between Single nucleotide polymorphisms and Eating in Atherosclerosis Susceptibility; CAD, coronary artery disease.

Data are expressed as mean and standard deviation or frequency (\%).

${ }^{\star} P$ values derived from Student's $t$ test or the $x^{2}$ test.

\section{Results}

A total of $999(81.8 \%)$ of the CAD patients were male and $222(18.2 \%)$ were female. On the other hand, 622 participants of the control group were male (46.3\%) and 722 $(53.7 \%)$ were female. The observed age difference between cases and controls was significant $(P=0 \cdot 001)$. The mean BMI value was also different between the two study groups $(P<0.05)$.

Table 1 summarizes the sociodemographic characteristics of the study's population. The control group reported more years of education than the group of cases $(P<0.001)$. A higher proportion of controls reported high levels of educational attainment and income in the upper economic classes than CAD patients $(P<0 \cdot 001)$.

The clinical characteristics and biochemical indices of the study's population are summarized in Table 2. A higher prevalence of the conventional established risk factors was observed in cases than in controls. Interestingly, a high proportion of controls had TC over $200 \mathrm{mg} / \mathrm{dl}$ with only $27.3 \%$ being treated with lipid-lowering agents. On the other hand, $80 \%$ of CAD patients were receiving hypolipidaemic medication.

Lifestyle characteristics are presented in Table 3. PAL was higher in controls when compared with cases $(P<0.001)$. A higher prevalence of smoking was recorded in $\mathrm{CAD}$ patients than in controls, with patients reporting
Table 2 Clinical characteristics and biochemical indices of the study participants; THISEAS study, 2006-2010

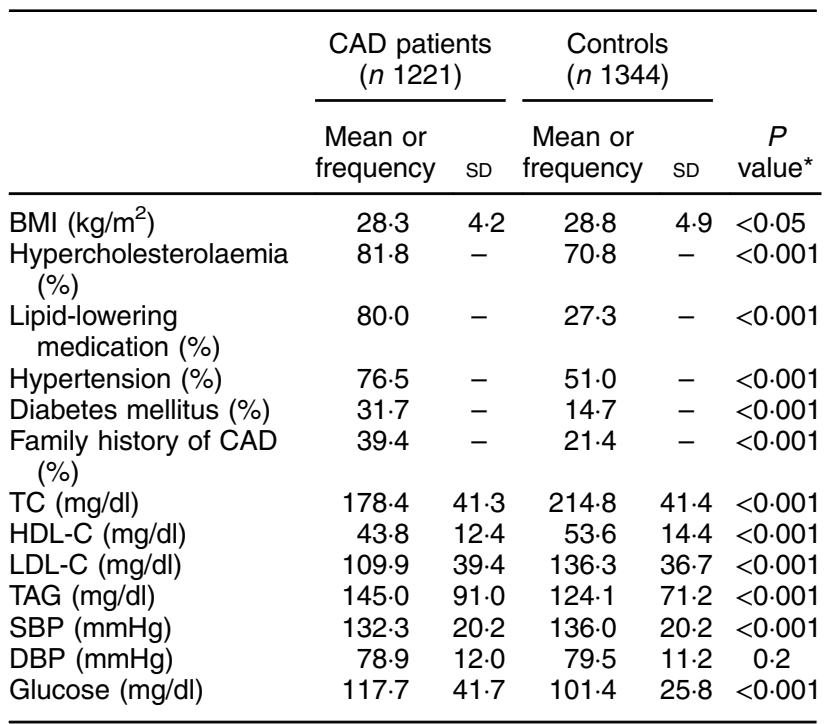

THISEAS, The Hellenic study of Interactions between Single nucleotide polymorphisms and Eating in Atherosclerosis Susceptibility; CAD, coronary artery disease; TC, total cholesterol; HDL-C, HDL cholesterol; LDL-C, LD cholesterol; SBP, systolic blood pressure; DBP, diastolic blood pressure. Data are expressed as mean and standard deviation or frequency (\%). ${ }^{*} P$ values derived from Student's $t$ test or the $X^{2}$ test.

almost double the pack-years of the control group $(P<0 \cdot 001)$. Concerning diet, the higher proportion of fat intake observed in the control group compared with cases was derived from the higher MUFA intake in the former study group $(P=0 \cdot 000)$. Exclusive use of olive oil in cooking was reported by a higher percentage of controls (11\%) compared with cases $(P<0.001)$.

After adjusting for conventional CAD risk factors, exclusive olive oil consumption was associated with $48 \%$ lower likelihood of developing CAD (OR $=0.52 ; 95 \% \mathrm{CI}$ $0 \cdot 35,0 \cdot 77 ; P=0 \cdot 001)$, as depicted in Table 4 . The previous finding was confirmed even after taking into account PA adoption and adherence to the MedDiet (OR $=0.63 ; 95 \%$ CI $0.42,0.93 ; P=0.02)$.

\section{Discussion}

The current work presents the design and descriptive characteristics of the THISEAS study. According to the study results, a higher prevalence of the established risk factors was observed in the group of cases. Higher values and above the normal range for TC and HDL-C were reported within the control group compared with cases. These results can be explained by the fact that most CAD patients were receiving lipid-lowering agents, mainly statins, while the majority of controls with TC values above $200 \mathrm{mg} / \mathrm{dl}$ were not receiving hypolipidaemic medication.

Moreover, almost $71 \%$ and $51 \%$ of the control group had hypercholesterolaemia and hypertension, respectively. The prevalence of hypercholesterolaemia within the control group is higher than previously reported in the Greek 
Table 3 Lifestyle characteristics of the study participants; THISEAS study, 2006-2010

\begin{tabular}{|c|c|c|c|c|c|}
\hline & \multicolumn{2}{|c|}{$\begin{array}{l}\text { CAD patients } \\
\text { (n 499) }\end{array}$} & \multicolumn{2}{|c|}{$\begin{array}{l}\text { Controls } \\
(n \text { 832) }\end{array}$} & \multirow[b]{2}{*}{$\begin{array}{c}P \\
\text { value* }\end{array}$} \\
\hline & $\begin{array}{l}\text { Mean or } \\
\text { frequency }\end{array}$ & SD & $\begin{array}{l}\text { Mean or } \\
\text { frequency }\end{array}$ & SD & \\
\hline $\begin{array}{l}\text { Current and } \\
\text { ex-smokers (\%) }\end{array}$ & $79 \cdot 3$ & - & $50 \cdot 6$ & - & $<0.001$ \\
\hline Pack-years & $54 \cdot 6$ & $42 \cdot 8$ & $28 \cdot 3$ & $26 \cdot 3$ & $<0.001$ \\
\hline PAL & $1 \cdot 3$ & 0.3 & 1.4 & 0.2 & $<0.001$ \\
\hline Energy intake (kJ) & 8820 & 3588 & 7665 & 3286 & $<0.001$ \\
\hline $\begin{array}{l}\text { Energy intake } \\
\quad(\mathrm{kcal})\end{array}$ & $2108 \cdot 0$ & $857 \cdot 6$ & 1831.9 & $785 \cdot 3$ & $<0.001$ \\
\hline Protein intake (\%E) & 17.5 & $3 \cdot 1$ & $19 \cdot 7$ & $4 \cdot 3$ & $<0.001$ \\
\hline $\begin{array}{l}\text { Carbohydrate } \\
\text { intake }(\% \mathrm{E})\end{array}$ & $45 \cdot 8$ & $8 \cdot 3$ & $44 \cdot 3$ & 9.6 & $<0.005$ \\
\hline Fat intake (\%E) & $35 \cdot 0$ & $6 \cdot 3$ & $36 \cdot 0$ & $6 \cdot 3$ & $<0.005$ \\
\hline SFA (\%E) & 11.6 & 3.5 & $11 \cdot 7$ & $3 \cdot 1$ & 0.7 \\
\hline MUFA (\%E) & $15 \cdot 4$ & $3 \cdot 2$ & $16 \cdot 4$ & $3 \cdot 8$ & $<0.001$ \\
\hline PUFA (\%E) & $5 \cdot 1$ & 1.3 & 4.9 & 1.5 & 0.1 \\
\hline $\begin{array}{l}\text { Exclusive olive oil } \\
\text { consumption (\%) }\end{array}$ & $65 \cdot 1$ & - & $76 \cdot 0$ & - & 0.001 \\
\hline
\end{tabular}

THISEAS, The Hellenic study of Interactions between Single nucleotide polymorphisms and Eating in Atherosclerosis Susceptibility; CAD, coronary artery disease; PAL, physical activity level; \%E, percentage of energy intake. Data are expressed as mean and standard deviation or frequency (\%).

${ }^{*} P$ values derived from Student's $t$ test or the $\chi^{2}$ test.

Table 4 Results from the logistic regression models for the evaluation of exclusive olive oil consumption on the risk of developing CAD in 259 CAD patients and 679 controls; THISEAS study, 2006-2010

\begin{tabular}{lccc}
\hline & OR & $95 \% \mathrm{Cl}$ & $P$ value \\
\hline Model 1* & 0.52 & $0.35,0.77$ & 0.001 \\
Model 2† & 0.62 & $0.42,0.92$ & 0.02 \\
Model 3 & 0.63 & $0.42,0.93$ & 0.02 \\
\hline
\end{tabular}

CAD, coronary artery disease; THISEAS, The Hellenic study of Interactions between Single nucleotide polymorphisms and Eating in Atherosclerosis Susceptibility.

*Model 1 included age, male sex, BMI, hypercholesterolaemia, hypertension, diabetes mellitus, family history of CAD and pack-years.

†Model 2 included model 1 and physical activity adoption.

¥Model 3 included model 2 and adherence to the Mediterranean diet.

population (ATTICA study), while the results regarding the prevalence of hypertension are similar to those reported previously ${ }^{(5)}$. However, the study sample in the THISEAS study is a decade older than the study aforementioned; therefore the results are not totally comparable.

In industrialized countries, socio-economic status is inversely related with CAD risk factors and CAD incidence, thus CAD mortality and morbidity is observed more among the less educated and less affluent ${ }^{(21,22)}$. Previous studies such as the Minnesota Heart Survey and the ATTICA study have demonstrated that low socioeconomic status can increase CAD risk factors or can predict unhealthy risk patterns ${ }^{(23,24)}$. Along the same lines, our results show that the control group reported higher education levels and higher financial status when compared with CAD patients.

$\mathrm{PA}$ is associated with lower incidence of $\mathrm{CAD}^{(25)}$. In our study, CAD patients recorded lower PAL than controls.
According to PAL values, CAD patients could be described as sedentary while controls could be described as physically active at low levels. Lower levels of PA in CAD patients than controls have also been reported in previous studies $^{(4,26)}$. These studies have also demonstrated a higher prevalence of current smoking and a higher number of pack-years in cases than controls, in line with our results.

Regarding dietary fat intake in both groups, the mean daily intakes of SFA and PUFA, as a percentage of total energy intake, are similar to those demonstrated for Greece by the EPIC (European Prospective Investigation into Cancer and Nutrition) study ${ }^{(27)}$. The proportion of total fat intake in the present study is lower than previously reported in the EPIC study for the Greek population but closer to the proposed guidelines ${ }^{(27,28)}$.

Olive oil is considered an important component of the MedDiet pattern and many studies have highlighted its inverse association with CAD occurrence ${ }^{(29,30)}$. The Greek population traditionally uses olive oil during meal and salad preparation. The beneficial effects of olive oil on CAD have been attributed to oleic acid and also to minor components, such as flavonoids, squalene and phenolic compounds ${ }^{(31)}$. The proposed mechanisms through which olive oil exerts its beneficial effects on CAD involve a decrease of TC and LDL-C, a reduction of LDL oxidation, an improvement of endothelial function and a decrease in thrombosis ${ }^{(11)}$.

In the present study, in addition to the well-established CAD risk factors, it is observed that the effect of exclusive olive oil consumption seems to play a significant role in CAD risk. Specifically, participants who reported exclusive use of olive oil during the preparation of meals and salads had $37 \%$ lower risk of developing CAD, regardless of their adherence to the MedDiet. This finding is similar to the results of prior studies conducted in the Greek population, namely the CARDIO2000 and the ATTICA studies ${ }^{(5,30)}$.

More recent prospective studies have shown that high olive oil consumption is inversely associated with CAD risk. In the EPICOR study, a reduction in CAD risk was observed among Italian women in the highest quartile of olive oil consumption during an approximately 8-year follow-up (hazard ratio $=0.56,95 \%$ CI $0.31,0.99 ; P=0.04)^{(32)}$. In the EPIC-Spain study, consumers in the highest quartiles of olive oil consumption had a $44 \%$ lower risk in CVD mortality compared with non-consumers ${ }^{(33)}$. The beneficial impact of olive oil consumption against CVD occurrence was further supported by the PREDIMED study, where a MedDiet supplemented with extra-virgin olive oil was associated with a reduction in CVD events (hazard ratio $=0.70$; CI 0.54 , $0 \cdot 92)^{(12)}$. Another study that examined olive oil consumption in the context of a MedDiet is the Three-City Study. That study's results revealed a lower stroke risk in high olive oil users, in elderly subjects. In agreement with our results, this outcome suggests that olive oil can be protective against disease regardless of other dietary elements ${ }^{(34)}$.

Although this finding regarding exclusive olive oil consumption may suggest an important approach to preventive 
nutrition, it does not suggest evidence for causality. Furthermore, it is worth mentioning that the quantity of olive oil intake was not assessed in the present work and there was no differentiation among different varieties of olive oil (common, virgin or extra-virgin olive oil).

Limitations of our case-control study are the selection and recall bias. A small percentage of future CAD patients may be wrongly assigned to the control group and recall bias may still exist regarding dietary information, smoking and PA habits. Nevertheless, we tried to eliminate both systematic errors as mentioned above (see 'Materials and methods' section).

Another important limitation is that there were differences in age, sex ratio and BMI between the two study groups resulting in an unmatched case-control study. However, we tried to control for these confounding factors during the logistic regression analysis of the study.

In addition, waist circumference data are not shown in our results due to the high number of missing values from CAD patients who were hospitalized and resting in bed during recruitment. Waist circumference is an independent predictor of CVD risk in overweight individuals ${ }^{(35,36)}$ and is in clinical utility in an attempt to track changes during lifestyle modifications even if BMI is not affected.

The main outcome of the present report is the finding that exclusive olive oil consumption in food and salad preparation can reduce the risk of CVD, even after controlling for potential confounders. Although many studies have demonstrated the beneficial effect of olive oil with regard to the variety or quantity consumed, not many studies have elucidated the effect of exclusive olive oil consumption. If this outcome is further confirmed, it suggests a simple, feasible and easily understood dietary recommendation for primary prevention of CVD occurrence.

\section{Conclusions}

The presented data provided the demographic, clinical and lifestyle characteristics of the THISEAS study. A higher prevalence of the established risk factors was reported among CAD patients. Exclusive olive oil consumption in cooking seems to lower the odds of CAD risk. This association remained significant even after taking into account CAD risk factors and lifestyle choices (PA and diet). However, further investigation is needed in larger sample sizes in order to elucidate the impact of exclusive olive oil consumption on CAD risk.

\section{Acknowledgements}

Acknowledgements: The authors thank all the dietitians and clinicians for their contribution to the project. Financial support: This work was partially supported by a research grant (PENED 2003) from the Greek General
Secretary of Research and Technology. The Greek General Secretary of Research and Technology had no role in the design, analysis or writing of this article. Conflict of interest: None. Authorship: M.D. participated in the data collection, carried out the data manipulation, analysis and interpretation, performed the statistical analysis and drafted the paper. L.S.R. contributed to the data collection and critically reviewed the paper. E.V.T. participated in the design of the study, participated in the data collection and critically reviewed the paper. I.P.K. participated in the data collection, data manipulation and critically reviewed the paper. G.K. contributed to the data collection and critically reviewed the paper. G.V.D. carried out the study design, supervised and coordinated the study and drafted the paper. All authors have approved the final article. Ethics of buman subject participation: The study protocol was approved by the Ethics Committee of Harokopio University.

\section{Supplementary material}

To view supplementary material for this article, please visit http://dx.doi.org/10.1017/S1368980015002244

\section{References}

1. World Health Organization (2011) Global Atlas on Cardiovascular Disease Prevention and Control. Geneva: WHO.

2. Aravanis C, Concordilas A, Dontas AS et al. (1970) Coronary heart disease in seven countries. IX. The Greek islands of Crete and Corfu. Circulation 4, 88-100.

3. Moulopoulos SD, Adamopoulos PN, Diamantopoulos EI et al. (1987) Coronary heart disease risk factors in a random sample of Athenian adults. The Athens Study. Am J Epidemiol 126, 882-892.

4. Panagiotakos DB, Pitsavos C, Chrysohoou C et al. (2002) Risk stratification of coronary heart disease in Greece: final results from the CARDIO2000 Epidemiological Study. Prev Med 35, 548-556.

5. Panagiotakos DB, Pitsavos C, Chrysohoou C et al. (2008) Five-year incidence of cardiovascular disease and its predictors in Greece: the ATTICA study. Vasc Med 13, 113-121.

6. Keys A (editor) (1970) Coronary heart disease in seven countries. Circulation 41, 1-211.

7. Keys A, Menotti A, Aravanis C et al. (1984) The seven countries study: 2,289 deaths in 15 years. Prev Med 13, 141-154.

8. Menotti A, Keys A, Aravanis C et al. (1989) Seven Countries Study. First 20-year mortality data in 12 cohorts of six countries. Ann Med 21, 175-179.

9. de Lorgeril M, Salen P, Martin JL et al. (1999) Mediterranean diet, traditional risk factors, and the rate of cardiovascular complications after myocardial infarction: final report of the Lyon Diet Heart Study. Circulation 16, 779-785.

10. Sofi F, Abbate R, Gensini GF et al. (2010) Accruing evidence on benefits of adherence to the Mediterranean diet on health: an updated systematic review and meta-analysis. Am J Clin Nutr 92, 1189-1196.

11. López-Miranda, Perez-Jimenez F, Ros E et al. (2010) Olive oil and health: summary of the II international conference on olive oil and health consensus report, Jaén and Córdoba (Spain) 2008. Nutr Metab Cardiovasc Dis 20, 284-294. 
12. Estruch R, Ros E, Salas-Salvadó J et al. (2013) Primary prevention of cardiovascular disease with a Mediterranean diet. $N$ Engl J Med 4, 1279-1290.

13. Huang CL \& Sumpio BE (2008) Olive oil, the Mediterranean diet, and cardiovascular health. J Am Coll Surg 207, 407-416.

14. Ferro-Luzzi A, James WP \& Kafatos A (2002) The high-fat Greek diet: a recipe for all? Eur J Clin Nutr 56, 796-809.

15. Kalra S, Duggal S, Valdez G et al. (2008) Review of acute coronary syndrome diagnosis and management. Postgrad Med 120, 18-27.

16. Friedewald WT, Levi RI \& Fredrickson DS (1972) Estimation of the concentration of low-density lipoprotein cholesterol in plasma, without use of the preparative ultracentrifuge. Clin Chem 18, 499-502.

17. Maraki M, Kollia A, Gioxari A et al. (2010) Development, reliability and validity of the Athens Physical Activity Questionnaire. Clin Nutr Suppl 5, 80.

18. Ainsworth BE, Haskell WL, Herrmann SD et al. (2011) 2011 Compendium of physical activities: a second update of codes and MET values. Med Sci Sports Exerc 43, 1575-1581.

19. Ministry of Health and Welfare (1999) Dietary guidelines for adults in Greece. Arch Hellenic Med 16, 516-524.

20. Panagiotakos DB, Pitsavos C, Arvaniti F et al. (2007) Adherence to the Mediterranean food pattern predicts the prevalence of hypertension, hypercholesterolemia, diabetes and obesity, among healthy adults; the accuracy of the MedDietScore. Prev Med 44, 335-340.

21. Marmot M (1989) Socioeconomic determinants of CHD mortality. Int J Epidemiol 18, 196-202.

22. Mackenbach JP, Kunst A, Cavelaars AE et al. (1997) Socioeconomic inequalities in morbidity and mortality in Western Europe. The EU Working Group on Socioeconomic Inequalities in Health. Lancet 349, 1655-1659.

23. Luepker RV, Rosamond W, Murphy R et al. (1993) Socioeconomic status and coronary heart disease risk factor trends. The Minnesota Heart Survey. Circulation 88, 2172-2179.

24. Panagiotakos DB, Pitsavos C, Chrysohoou C et al. (2008) The effect of clinical characteristics and dietary habits on the relationship between education status and 5-year incidence of cardiovascular disease: the ATTICA study. Eur J Nutr $\mathbf{4 7}$, $258-265$.
25. Thompson PD, Buchner D, Piña IL et al. (2003) AHA Scientific Statement. Exercise and physical activity in the prevention and treatment of atherosclerotic cardiovascular disease. Circulation 107, 3109-3116.

26. Gustavsson J, Mehling K, Leander K et al. (2015) FTO gene variation, macronutrient intake and coronary heart disease risk: a gene-diet interaction analysis. Eur $J$ Nutr (Epublication ahead of print version).

27. Linseisen J, Welch AA, Ocké M et al. (2009) Dietary fat intake in the European Prospective Investigation into Cancer and Nutrition: results from the 24-h dietary recalls. Eur J Clin Nutr 63, Suppl. 4, S61-S80.

28. International Task Force for Prevention of Coronary Heart Disease (2003) Pocket Guide to Prevention of Coronary Heart Disease. Grünwald: Börm Bruckmeier.

29. Fernández-Jarne E, Martinez-Losa E, Prado-Santamaría M et al. (2002) Risk of first non-fatal myocardial infarction negatively associated with olive oil consumption: a casecontrol study in Spain. Int J Epidemiol 31, 474-480.

30. Kontogianni MD, Panagiotakos DB, Chrysohoou C et al. (2007) The impact of olive oil consumption pattern on the risk of acute coronary syndromes: the CARDIO2000 case-control study. Clin Cardiol 30, 125-129.

31. Waterman E \& Lockwood B (2007) Active components and clinical applications of olive oil. Altern Med Rev 12, 331-342.

32. Bendinelli B, Masala G, Saieva C et al. (2011) Fruit, vegetables, and olive oil and risk of coronary heart disease in Italian women: the EPICOR Study. Am J Clin Nutr 93, 275-283.

33. Buckland G, Mayén AL, Agudo A et al. (2012) Olive oil intake and mortality within the Spanish population (EPIC-Spain). Am J Clin Nutr 96, 142-149.

34. Samieri C, Féart C, Proust-Lima C et al. (2011) Olive oil consumption, plasma oleic acid, and stroke incidence: the Three-City Study. Neurology 77, 418-425.

35. Lofgren I, Herron K, Zern T et al. (2004) Waist circumference is a better predictor than body mass index of coronary heart disease risk in overweight premenopausal women. $J$ Nutr 134, 1071-1076.

36. Freiberg MS, Pencina MJ, D'Agostino RB et al. (2008) BMI vs. waist circumference for identifying vascular risk. Obesity (Silver Spring) 16, 463-469. 